\title{
Bulk dynamics for interfacial growth models
}

\author{
Cristóbal López, ${ }^{1}$ Pedro L. Garrido, ${ }^{2}$ and Francisco de los Santos ${ }^{3}$ \\ ${ }^{1}$ Instituto Mediterráneo de Estudios Avanzados, CSIC-Universitat de les Illes Balears, E-07071 Palma de Mallorca, Spain \\ ${ }^{2}$ Instituto Carlos I de Física Teórica y Computacional, Universidad de Granada, E-18071 Granada, Spain \\ ${ }^{3}$ Centro de Física da Matéria Condensada da Universidade de Lisboa, Avenida Professor Gama Pinto 2, \\ P-1649-003 Lisboa, Portugal
}

(Received 18 May 2000)

We study the influence of the bulk dynamics of a growing cluster of particles on the properties of its interface. First, we define a general bulk growth model by means of a continuum Master equation for the evolution of the bulk density field. This general model just considers an arbitrary addition of particles (though it can be easily generalized to consider subtraction) with no other physical restriction. The corresponding Langevin equation for this bulk density field is derived where the influence of the bulk dynamics is explicitly shown. Finally, when a well-defined interface is assumed for the growing cluster, the Langevin equation for the height field of this interface for some particular bulk dynamics is written. In particular, we obtain the celebrated Kardar-Parisi-Zhang equation. A Monte Carlo simulation illustrates the theoretical results.

PACS number(s): 05.40.-a, 05.70.Ln, 68.35.Ct, 68.35.Fx

\section{INTRODUCTION}

In the last 15 years there has been a great interest in the study of the growth of surfaces by dynamic processes based on the addition and subtraction of particles (see, for example, [1-4]). For instance, the understanding of the conditions under which a growing surface shows a rough structure is nowadays of the greatest importance in the production of thin films and/or pure crystals. Surface growth is usually studied by using lattice models in which simple stochastic rules intend to mimic the relevant phenomena. Their extensive computer simulation have been of a major importance in characterizing and understanding the different shapes that occur in real experiments. However, due to the intrinsic limitations of computers capabilities, some very interesting aspects are usually subject to incomplete analysis and data interpretation. In particular, let us remark on the inherent difficulty in the study of the surface long-time behavior and its scaling properties. Nevertheless, for this particular aspect, analytical models seem to give us the answers to the questions that the computers fail to clarify. These are mainly based in postulating a Langevin equation for the height of the interface measured from a reference substrate. Such Langevin equations intend to mimic the system microscopic dynamics and its collective effect at large scales in space and time. A general choice has the structure

$$
\partial_{t} h_{t}(\mathbf{x})=\nu_{1} \nabla^{2} h_{t}+\nu_{2}\left|\nabla h_{t}\right|^{2}+\nu_{3} \nabla^{2} \nabla^{2} h_{t}+\cdots+\eta_{t}(\mathbf{x}),
$$

where $h_{t}(\mathbf{x})$ is the height of the interface at time $t$ at the substrate position $\mathbf{x} \in \mathbb{R}^{d}$ and $\eta_{t}(\mathbf{x})$ is a white noise term. Generally, a one-to-one correspondence is assumed between various terms in Eq. (1.1) and different physical processes (for example see the discussion in [5] concerning a model for epitaxial growth, or a general method in [6] to propose an equation such as Eq. (1.1) by using the reparametrization invariance symmetry). The details of the microscopic processes that are assumed to be irrelevant at this scale of ob- servation are taken into account through the values of the coefficients $\nu_{1}, \nu_{2}, \ldots$ and the properties of the noise term $\eta_{t}$. Once the Langevin equation (1.1) is defined, one may apply renormalization-group procedures to obtain different universal properties and scaling behaviors. The success of this scheme is clearly represented by the definition and analysis of the Kardar-Parisi-Zhang equation (KPZ) [7] which has been a clear breakthrough in the study of the space-time asymptotic behavior of growth models.

Quite often surface growth is a consequence of bulk dynamic processes. A good example of this is provided by the growth of bacterial colonies where bacteria multiply in a nutrient environment, the shape of the colony being the moving interface [8]. In general, the dynamics of the particles before and after its aggregation to a substrate may influence the system interfacial behavior. For instance, shadowing effects may appear as happens in diffusion limited aggregation (DLA) processes and in thin-film growth [9], or they may induce different scaling regimes depending on the time interval studied as it is shown in some molecular-beam-epitaxy models in which a system bulk dynamics is defined [10,11]. However, interface models are usually expressed in terms of a height field, $h_{t}(\mathbf{x}) \geqslant 0$. In doing so, bulklike contributions are neglected since only interfacial degrees of freedom are being considered. Unfortunately, the mathematical hurdles to deriving the phenomenological dynamics of interfaces from stochastic bulk microscopic models is formidable, and a comprehensive theoretical picture is still lacking although a significant body of rather rigorous work has been devoted to the subject [12].

It is well known that the macroscopic behavior of systems at nonequilibrium states exhibits a strong dependence on the functional structure of its microscopic dynamics (for instance, in the so-called two-temperature Ising model [13] one finds that the phase diagram changes radically depending on the analytic form for the probability of a spin flip). Nevertheless, one may expect that this strong relation between microscopic dynamics and macroscopic behavior should disappear near a renormalization-group (RG) fixed point or in the scaling regime where universality seems to guarantee that the 


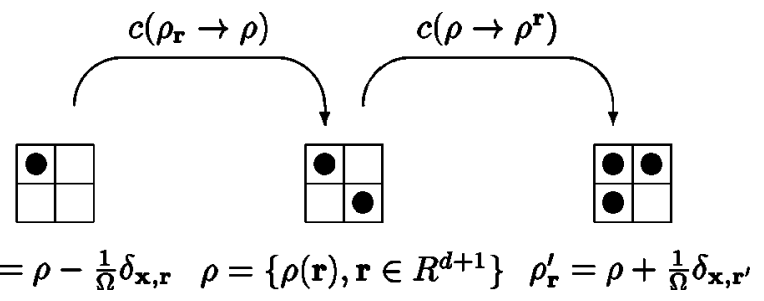

FIG. 1. Schematic representation of the process described by the Master equation (2.1).

microscopic details are irrelevant (at least, one knows that this is true when studying the dynamic properties of equilibrium systems near a (RG) fixed point [14]). However, some recent results on the critical behavior of a nonequilibrium driven diffusive system show that the microscopic dynamics may play a relevant role in the determination of the system universality class [15]. The influence of the microscopic dynamical details into the critical and noncritical properties of a nonequilibrium model implies, in our opinion, that any $a$ priori construction of a Langevin equation as Eq. (1.1) may occasionally disregard important features.

In this paper we introduce a quite general class of nonequilibrium bulk growth models for which the aforementioned problems can be addressed (there are in the literature some efforts in this direction [16-18]). We define a stochastic bulk local dynamics expressed by an appropriated continuum Master equation in which, for simplicity, only addition of particles is considered. From the Master equation and using a truncated Kramers-Moyal expansion, we derive a Langevin equation for the bulk degrees of freedom in which there is an explicit dependence on the analytic form of the rates. In order to study surface properties, in a subsequent section we derive, from this bulk equation, an expression for the interfacial height field dynamics. The illustrative example we take is that of the KPZ equation and, for consistency, in that particular case we check our results by means of a Monte Carlo simulation. Some other examples are then briefly commented on and our conclusions are given in the final section.

\section{GROWTH MODELS: A GENERAL DESCRIPTION}

Let us consider a particle density field, $\rho(\mathbf{x}, \tau), \mathbf{x} \in \mathbb{R}^{d+1}$, and assume that the probability distribution $P_{\tau}(\rho)$ associated with each field configuration obeys the following continuous Master equation:

$$
\partial_{\tau} P_{\tau}(\rho)=\int_{R^{d+1}} d \mathbf{r}\left[c\left(\rho_{\mathbf{r}} \rightarrow \rho\right) P_{\tau}\left(\rho_{\mathbf{r}}\right)-c\left(\rho \rightarrow \rho^{\mathbf{r}}\right) P_{\tau}(\rho)\right] .
$$

Here, $c\left(\rho \rightarrow \rho^{\prime}\right)$ is the probability per unit time (or transition rate) from one configuration $\rho$ to another $\rho^{\prime}$, and $\rho_{\mathbf{r}}=\rho(\mathbf{x})$ $-\Omega^{-1} \delta(\mathbf{x}-\mathbf{r}), \rho^{\mathbf{r}}=\rho(\mathbf{x})+\Omega^{-1} \delta(\mathbf{x}-\mathbf{r})$. Note that the density field, $\rho$, can only grow in steps of size $\Omega^{-1}$. This is consistent with a picture in which $\rho(\mathbf{r})$ is a particle density that results after coarse graining over blocks of size $\Omega$ centered around $\mathbf{r}$ in a lattice. Therefore, the Master equation (2.1), so defined, could be thought of as if it only described processes that add one particle per block of the lattice per elementary time step. This is represented in Fig. 1.
Next, we choose the transition rates such that

$$
c\left(\rho \rightarrow \rho^{\prime}\right)=w(\rho ; \mathbf{r})
$$

namely, they are a function that depends only on the initial configuration $\rho$ and the specific point where mass is added. Now, let us assume that $\Omega$ is large enough (typically, it should be much bigger than any microscopic length scale present in the original physical problem but much smaller than the correlation length of the system) and expand the Master equation (2.1) on inverse powers of $\Omega$. Then, using the expansion

$$
\begin{gathered}
c\left(\rho \rightarrow \rho^{\mathbf{r}}\right)=w(\rho ; \mathbf{r}), \\
c\left(\rho_{\mathbf{r}} \rightarrow \rho\right)=w\left(\rho-\Omega^{-1} \delta(x-r) ; \mathbf{r}\right) \\
=\sum_{m=0}^{\infty} \frac{(-1)^{m}}{m ! \Omega^{m}} \frac{\delta^{m}}{\delta \rho(\mathbf{r})^{m}} w(\rho ; \mathbf{r}), \\
P_{\tau}\left(\rho_{\mathbf{r}}\right)=\sum_{m=0}^{\infty} \frac{(-1)^{m}}{m ! \Omega^{m}} \frac{\delta^{m}}{\delta \rho(\mathbf{r})^{m}} P_{\tau}(\rho),
\end{gathered}
$$

we get the so-called Kramers-Moyal expansion of the Master equation (2.1) [19],

$$
\partial_{\tau} P_{\tau}(\rho)=\int_{R^{d+1}} d \mathbf{r} \sum_{l=1}^{\infty} \frac{(-1)^{l}}{l ! \Omega^{l}} \frac{\delta^{l}}{\delta \rho(\mathbf{r})^{l}}\left[w(\rho ; \mathbf{r}) P_{\tau}(\rho)\right] .
$$

The next step we take is to keep only the first two terms in Eq. (2.6). Then, we can write down the following FokkerPlanck equation:

$$
\begin{aligned}
\partial_{\tau} P_{\tau}(\rho)= & \int_{R^{d+1}} d \mathbf{r}\left[-\frac{1}{\Omega} \frac{\delta}{\delta \rho(\mathbf{r})}+\frac{1}{2 \Omega^{2}} \frac{\delta^{2}}{\delta \rho(\mathbf{r})^{2}}\right] \\
& \times\left[w(\rho ; \mathbf{r}) P_{t}(\rho)\right] .
\end{aligned}
$$

To control the goodness of such an approximation we appeal to the Kurtz theorem [20], by virtue of which when $\Omega \rightarrow \infty$, and for a given time $T<\infty$ then

$$
\sup _{\tau<T}\left|\rho_{\tau}-\tilde{\rho}_{\tau}\right| \leqslant \zeta_{\Omega}^{T} \frac{\ln \Omega}{\Omega}
$$

where $\rho_{\tau}$ and $\tilde{\rho}_{\tau}$ are typical time trajectories on phase space which are solutions of the exact Master equation (2.1) and the Fokker-Planck one (2.7), respectively. $\zeta_{\Omega}^{T}$ is a random variable whose distribution does not depend on $\Omega$ and satisfying $\left\langle\exp \left(\lambda \zeta_{\Omega}^{T}\right)\right\rangle<\infty$ for any constant $\lambda>0$. That is, for a given fixed time $T$ one can always find a large enough $\Omega$ such that the difference between solving exactly the master equation or solving the truncated version of it, is of order $\ln \Omega / \Omega$ and, therefore, negligible. Moreover, this bound is the best one and no new terms of the Kramers-Moyal expansion give better results. However, when $T \rightarrow \infty$ one cannot control, in general, the accumulated influence of the neglected terms in the expansion. But, since the study of growth models is mainly focused on the understanding of 
their evolution properties, due to Kurtz's theorem the Fokker-Planck equation (2.7) is a valid theoretical starting point.

Last, the Fokker-Planck equation (2.7) is equivalent in the Stratonovich sense to the Langevin equation [19]

$$
\begin{aligned}
\partial_{t} \rho(\mathbf{r}, t)= & w(\rho ; \mathbf{r})-\frac{1}{4 \Omega} \frac{\delta}{\delta \rho(\mathbf{r})} w(\rho ; \mathbf{r}) \\
& +\frac{1}{\Omega^{1 / 2}} w(\rho ; \mathbf{r})^{1 / 2} \nu_{t}(\mathbf{r}),
\end{aligned}
$$

where $t=\Omega^{-1} \tau, \nu_{t}(\mathbf{r})$ is a white noise with zero mean and $\left\langle\nu_{t}(\mathbf{r}) \nu_{t^{\prime}}\left(\mathbf{r}^{\prime}\right)\right\rangle=\delta\left(\mathbf{r}-\mathbf{r}^{\prime}\right) \delta\left(t-t^{\prime}\right)$. We recall that $w(\rho ; \mathbf{r})$ is the probability per unit time of adding a particle (of mass $\Omega^{-1}$ ) at point $\mathbf{r}$. If we considered the possibility of particle subtractions then we should do the following substitutions in Eq. (2.9): $w \rightarrow w_{+}-w_{-}$in the first term and $w \rightarrow w_{+}+w_{-}$in the last two terms. $w_{+(-)} \equiv w(\rho ; \mathbf{r})_{+(-)}$is the probability per unit time of adding (subtracting) a particle.

Before we proceed to the next section, let us remark that (a) Eq. (2.9) describes the evolution of the bulk density field of a system that growths by addition of particles with, in principle, no other physical assumptions, and (b) the Langevin equation (2.9) depends directly on the functional form of the bulk rates. The election of these bulk rates then provides the physical restrictions for the particular growth model that is going to be specifically modeled. Also, it is remarkable that the influence of the bulk dynamics affects the noise term through a nontrivial factor.

\section{HEIGHT DYNAMICS: KPZ EQUATION AND MONTE CARLO SIMULATION}

We proceed to single out the interfacial degrees of freedom of Eq. (2.9). In order to achieve this, we place two conditions on the solutions of Eq. (2.9). First, let us impose that our bulk dynamics produces a surface perpendicular to the $z$ axis without overhangs and bubbles. This condition is necessary to ensure that we have a well defined interface [note that Eq. (2.9) contains overhang-vacancy and shadowing effects]. Second, we neglect any interfacial profile. Then, we may assume that the solutions of the Langevin equation (2.9) have the form

$$
\rho(\mathbf{r}, t)=\Theta\left(h_{t}(\mathbf{x})-z\right),
$$

where $\mathbf{r} \equiv(\mathbf{x}, z), \mathbf{x} \in \mathbb{R}^{d}$ is a point in the substrate and $h_{t}(\mathbf{x})$ is the height of the growing surface at time $t . \Theta(\lambda)=1,1 / 2,0$ if $\lambda>0,=0,<0$, respectively. That is, for a given point in the bulk $\mathbf{r}$, if its $z$ coordinate is larger, equal or smaller than the actual position of the surface, $h_{t}(\mathbf{x})$, then the density field is $\rho(\mathbf{r}, t)=0,1 / 2$ and 1 , respectively. Note that since the $\Theta$ function is not continuous, when differentiating we should use a regularized version of it, e.g., $\Theta(x)=\frac{1}{2}[1+\tanh (a x)]$ with $a \rightarrow \infty$.

We are interested in constructing a dynamical equation for the $h_{t}(\mathbf{x})$ fields. Therefore, let us make a time derivative in Eq. (3.1)

$$
\partial_{t} \rho(\mathbf{r}, t)=\partial_{t} h_{t}(\mathbf{x}) \delta\left(h_{t}(\mathbf{x})-z\right) .
$$

Integrating in $z$ both sides of Eq. (3.2) we find

$$
\partial_{t} h_{t}(\mathbf{x})=\int_{R} d z \quad \partial_{t} \rho(\mathbf{r}, t) .
$$

Equating this expression for $\partial_{t} h_{t}(\mathbf{x})$, together with Eq. (2.9), will lead us to the desired Langevin equation for the heights.

To make this a bit more concrete, we now introduce a particular set of rates. For instance, we choose the probability of adding mass to the point $\mathbf{r}$ to be proportional to the square of the gradient of the density field in that point: $w(\rho ; \mathbf{r}) \propto|\nabla \rho|^{2}$. With this election the unwanted effect of nucleation of bubbles is avoided. After a bit of algebra, we get

$$
\partial_{t} h_{t}=\alpha\left[1+\left(\nabla h_{t}\right)^{2}\right]+\frac{D}{2 \Omega} \Delta h+\frac{1}{\Omega^{1 / 2}}\left[1+\left(\nabla h_{t}\right)^{2}\right]^{1 / 2} \nu_{t},
$$

which is the celebrated KPZ equation with a different noise term [a naive power counting argument reduces the relevant part of Eq. (3.4) to the KPZ equation]. The coefficient $D$ has the proper dimensions and $\alpha$ is positive and depends on how the $\Theta$ function is regularized. This comes from our particular ansatz, but, we would like to stress here that, as far as universal properties are concerned, the precise value of the coefficients is immaterial. In fact, it is easy to show (with naive power counting) that for any bulk dynamics given by $w(\rho ; r) \propto|\nabla \rho|^{\eta}$ with $\eta \geqslant 0$, gives rise to a height equation falling in the KPZ universality class.

Next, we proceed to check numerically the connection between Eq. (2.1) with $w(\rho ; \mathbf{r}) \propto|\nabla \rho|^{2}$ and the KPZ equation (3.4).

Numerical results. The simple bulk rate $|\nabla \rho|^{2}$ can be easily implemented in a Monte Carlo experiment. On a twodimensional square lattice periodic boundary conditions are considered in one of the principal axes. Each lattice site is labeled by an occupation variable $\rho_{\mathbf{r}}$ ranging from $0,1 / \Omega, \ldots$ to 1 . A site is empty if $\rho_{\mathbf{r}}=0$ and full if $\rho_{\mathbf{r}}=1$. Initially the lattice is empty except for a full horizontal bottom line. The growth starts when an empty site $\mathbf{r}$ is chosen at random from the lattice. Then, according to Eq. (2.1) and $w \propto|\nabla \rho|^{2}, \rho_{\mathbf{r}}$ is increased in $\Omega^{-1}$ with a probability that depends on each nearest neighbor of $\mathbf{r}$ through $\left|\nabla \rho_{\mathbf{r}}\right|^{2}$. The next site is selected at random from those who are empty or partially filled. It should be noted that neither filled sites, $\rho_{\mathbf{r}}=1$, nor sites in the vacuum phase, i.e., sites surrounded by empty nearest neighbors, can grow. Thus, if we define the height $h_{t}(x)$ as the distance to the highest (fully or partially) occupied lattice site directly above the substrate coordinate $x$, we conclude that growth is restricted to the bulk phase or to the vicinity of the interface. Nucleation of droplets in the empty phase is not possible. In Fig. 2 we show a snapshot of the growing interface for $\Omega=15$. Darker regions correspond to higher densities. The bulk sites below the interface which are not filled are still evolving and determine the future evolution of the interface.

Numerically, one has to be very careful with the use of a centered-in-space form for the discrete gradient operator, since this may give rise to bulk vacancies incompatible with Eq. (3.1). That is, a nonfull lattice site with full nearest 


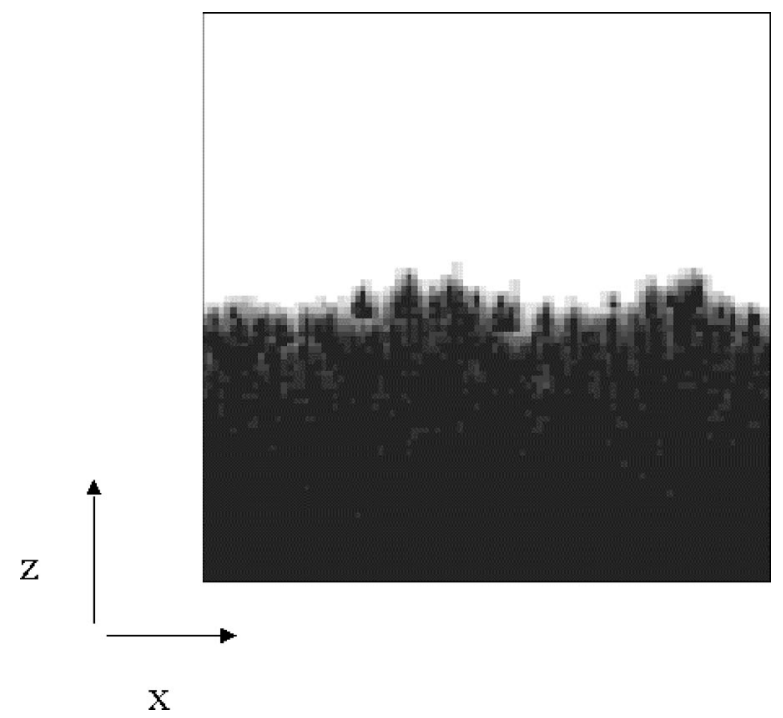

FIG. 2. Snapshot of a growing interface. The greyscale ranges from white (empty site) to black (full site).

neighbors can never be updated. This leads to voids in the bulk phase in contradiction with our assumption of the absence of bubbles. Therefore, we use the following finitedifference formula for the density derivatives $[\mathbf{r}=(x, z)]$

$$
\nabla \rho_{\mathbf{r}}=(\rho(x \pm 1, z)-\rho(x, z), \rho(x, z)-\rho(x, z-1)),
$$

where left and right derivatives are used alternately to avoid asymmetric effects and, for convenience, a unit lattice spacing is assumed. With this finite-difference scheme such inert points into the bulk phase are no longer observed.

Figure 3 shows the scaling plot for the surface width $W(L, t)^{2}=L^{-1} \Sigma\left[h_{t}(x)-\bar{h}(t)\right]^{2}$ for different system substrate sizes $L . \bar{h}(t)$ is the mean height of the interface at time $t$. The numerical data were averaged over 2000 independent runs for $L=100,200,500$, and over 1000 independent runs for $L=800$. Different values of $\Omega$ yield similar results for the particular dynamics given by $\left|\nabla \rho_{\mathrm{r}}\right|^{2}$. But it cannot be guaranteed that this will be the case for other types of rates. Recall that $\Omega$ is a length scale that must be bigger than any microscopic length scale. In fact $\Omega=1$ is the limit of vanishing bulk's influence on the interface. Here, for reasons of computational efficiency the results shown correspond to $\Omega$

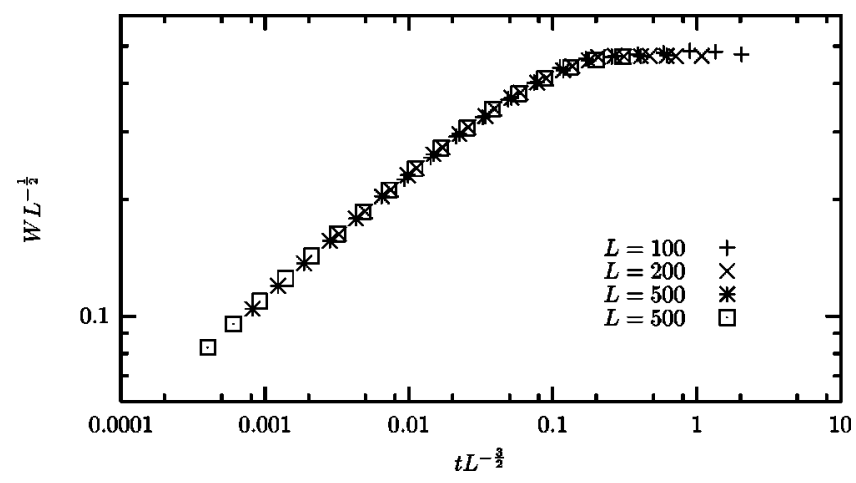

FIG. 3. Scaling plot for the model defined by the Master equation (2.1) with the rate $|\nabla \rho|^{2}$. The data are for $L=100,200,500$, and 800 , where $L$ is the width of the substrate.
$=1$. Good data collapse is obtained for a roughness exponent $\alpha=1 / 2$ and a dynamic exponent $z=3 / 2$, in agreement with the KPZ prediction [7].

The example we have just provided is by no means unique. Our formalism encompasses many other well-known growth equations. Let us just mention that with the simple dynamics given by $w(\rho, \mathbf{r})=|\nabla \rho|$ the equation of Golubovic and Wang, related to the anharmonic equilibrium thermal fluctuations of smectic-A phases [21], is obtained. This is given by

$$
\begin{aligned}
\partial_{t} h_{t}= & {\left[1+\left(\nabla h_{t}\right)^{2}\right]\left(\lambda+\frac{1}{\Omega} H\right) } \\
& +\frac{1}{\Omega^{1 / 2}}\left[1+\left(\nabla h_{t}\right)^{2}\right]^{1 / 4} \nu_{t},
\end{aligned}
$$

where $\lambda$ is a coefficient and $H$ is the curvature (see [21]). As we have mentioned before, this kind of dynamics (proportional to $|\nabla \rho|^{\eta}$ with $\eta \geqslant 0$ ), falls in the KPZ universality class. Also, the Edwards-Wilkinson equation [22], which favors growth at local minima, can also be recovered by considering subtraction of particles and a rate of the form $w$ $=\left|\nabla^{2} \rho\right|$. In this case, the formalism has to be slightly modified by linking the election of addition or subtraction of particles to the density field configuration. More explicitly, now the Master equation defining the process reads

$$
\partial_{\tau} P_{\tau}(\rho)=\int_{R^{d+1}} d \mathbf{r}\left[c\left(\rho^{\prime} \rightarrow \rho\right) P_{\tau}\left(\rho^{\prime}\right)-c\left(\rho \rightarrow \rho^{\prime}\right) P_{\tau}(\rho)\right]
$$

with $\rho^{\prime}=\rho(\mathbf{x})+\alpha \Omega^{-1} \delta(\mathbf{x}-\mathbf{r})$ and $\alpha=-1,0,1$ for $\nabla^{2} \rho$ less, equal and greater than 0 , respectively. That is, material is added to those areas where the Laplacian of the density field is negative and taken from those where it is positive. In this manner, a balanced distribution of mass is achieved that results in the equilibrium Edwards-Wilkinson universality class.

Many other different rates lead to their corresponding growth equations, sometimes to the same one, showing that growth models with similar surface behavior may not have the same bulk properties.

\section{CONCLUSIONS}

In this paper we have introduced a class of nonequilibrium models in which a stochastic bulk dynamics is defined. The bulk evolves by an absorption process represented by a continuum Master equation. From it, we have derived a Langevin equation for the bulk density field whose structure depends on the details of the underlying bulk dynamics. This dependence was then extended to an equation of motion for the interfacial degrees of freedom. In particular, we have exemplified the procedure by deriving the KPZ equation from a very simple bulk rate. A Monte Carlo simulation of 
the bulk process confirms the predicted scaling behavior for the interface. Finally, a number of examples were briefly mentioned. In all cases the bulk dynamics determines the mesoscopic height equation, showing that both scales are related in a nontrivial form and that their mutual influence could be far from intuitive.

The strategy developed in this paper is quite general. It includes both local and nonlocal, and equilibrium and nonequilibrium growth processes. Therefore, a great number of growth physical phenomena can be studied, in principle, with our approach. For instance, molecular-beam-epitaxy models with adatom mobility, driven lattice-gases or wetting phenomena by means of lattice gas theories of multilayer adsorption, to name just a few.

\section{ACKNOWLEDGMENTS}

We acknowledge E. Hernández-García and M.A. Muñoz for a critical reading of the manuscript. C.L. acknowledges the hospitality received at the Centro de Física da Matéria Condensada da Universidade de Lisboa and is supported by CICYT (MAR98-0840). F.S. was supported by the European Commission under Grant No. ERBFMRXCT980183.
[1] J. Krug and H. Spohn in Solids far from Equilibrium, edited by C. Godreche (Cambridge University Press, Cambridge, 1992).

[2] J. Kertész and T. Vicsek, in Self-affine Interfaces in Fractals in Science, edited by A. Bunde and S. Havlin (Springer-Verlag, Berlin, 1994), p. 89.

[3] A.L. Barabasi and H.E. Stanley, Fractal Concepts in Surface Growth (Cambridge University Press, Cambridge, 1995).

[4] T. Halpin-Healy and Y.C. Zhang, Phys. Rep. 254, 215 (1995).

[5] Z.W. Lai and S.D. Sarma, Phys. Rev. Lett. 66, 2348 (1991).

[6] M. Marsili, A. Maritan, F. Toigo, and J.R. Banavar, Rev. Mod. Phys. 68, 963 (1996).

[7] M. Kardar, G. Parisi, and Y.C. Zhang, Phys. Rev. Lett. 56, 889 (1986).

[8] T. Vicsek, M. Cserzö, and V.K. Horváth, Physica A 167, 315 (1990).

[9] R.P.U. Karunasiri, R. Bruinsma, and J. Rudnick, Phys. Rev. Lett. 62, 788 (1989).

[10] S. Das Sarma, C.J. Lanczycki, S.V. Ghaisas, and J.M. Kim, Phys. Rev. B 49, 10693 (1994).

[11] M. Schimschak and J. Krug, Phys. Rev. B 52, 8550 (1995).
[12] H. Spohn, J. Stat. Phys. 71, 1081 (1993).

[13] P.L. Garrido and J. Marro, J. Phys. A 25, 1453 (1992); J. Stat. Phys. 74, 663 (1994).

[14] P.C. Hohenberg and B.I. Halperin, Rev. Mod. Phys. 49, 435 (1977).

[15] P.L. Garrido, M.A. Muñoz, and F. de los Santos, Phys. Rev. E 61, R4683 (2000).

[16] B. Grossmann, H. Guo, and M. Grant, Phys. Rev. A 43, 1727 (1991).

[17] P. Keblinski, A. Maritan, F. Toigo, and J.R. Banavar, Phys. Rev. E 49, R4795 (1994).

[18] G. Parisi and Y.C. Zhang, J. Stat. Phys. 41, 1 (1985).

[19] N.G. van Kampen, Stochastic Processes in Physics and Chemistry (North-Holland, Amsterdam, 1992).

[20] T.G. Kurtz, Stoch. Proc. Appl. 6, 223 (1978).

[21] L. Golubovic and Z.G. Wang, Phys. Rev. Lett. 69, 2535 (1992).

[22] S.F. Edwards and D.R. Wilkinson, Proc. R. Soc. London, Ser. A 381, 17 (1982). 